Zeitschriftenartikel:

Begutachtet

Redaktion und Begutachtung:

Nele Heise (iD)

Digital Media \& Communication

Researcher Hamburg

Nils Zurawski (DD

Universität Hamburg

Erhalten: 01. Dezember 2019

Akzeptiert: 29. September 2020

Publiziert: 15. Dezember 2020

Lizenz:

(c) Lena Wiesler

Dieses Werk steht unter einer Lizenz

Creative-Commons-Namensnennung 4.0

(CC-BY 4.0) International

(C) (i)

Datenverfügbarkeit:

Alle relevanten Daten befinden sich innerhalb der Veröffentlichung.

Interessenskonfliktstatement:

Die Autor:innen erklären, dass ihre Forschung ohne kommerzielle oder finanzielle Beziehungen durchgeführt wurde, die als potentielle

Interessenskonflikte ausgelegt werden können.

\section{Empfohlene Zitierung:}

Wiesler, L. (2020). Kritische Stimmen im Netz: Audio-Podcasting als feministische Praxis.kommunikation@gesellschaft, 21(2). https://doi.org/10.15460/kommges. 2020.21.2.625

\section{Kritische Stimmen im Netz}

\section{Audio-Podcasting als feministische Praxis}

\author{
Lena Wiesler ${ }^{a *}$ iD \\ ${ }^{a}$ Freie Medienpädagogin, Heidelberg \\ * Korrespondenz: lenawiesler@posteo.de
}

\begin{abstract}
Feministische Podcasts werden in diesem Beitrag als vernetzte Gegenöffentlichkeiten verstanden und der Aktivismus feministischer Podcaster:innen als kollektive und vernetzte feministische Praxis. Im Zentrum der explorativen Studie stehen die Praktiken unabhängiger feministischer Podcaster:innen, welche durch offene leitfadengestützte Interviews mit sechs Podcasterinnen aus Deutschland und Österreich ergründet werden. Die Ergebnisse können anhand von drei Kernkategorien zusammengefasst werden: erstens das Verständnis von Feminismus als Reflexionsund Aushandlungsprozess, zweitens feministisches Podcasting als Ausdruck von Zugehörigkeit und Unabhängigkeit sowie drittens Austausch und Vernetzung als Kernpraxis feministischer Podcaster:innen.
\end{abstract}

Schlagworte: Podcasting, Feminismus, feministische Medien, Netzfeminismus, unabhängige Podcasts, feministische Praxis 


\section{Einleitung}

In der Vielfalt feministischer Medien findet sich in den letzten Jahren eine stetig wachsende Zahl an Podcasts, die feministische Perspektiven und Identitäten hörbar machen und Geschlechterverhältnisse in Frage stellen. Feministische Podcaster:innen sind damit Teil einer Reihe feministischer Aktivist:innen, die die partizipativen Potentiale von Medien nutzen, um eigene Kommunikationsräume und Gegenöffentlichkeiten zu schaffen abseits hegemonialer Diskurse und klassischer Massenmedien. Insbesondere der Einsatz digitaler Medien und die Nutzung des Internets haben in den letzten Jahrzehnten die Möglichkeiten zur Verbreitung feministischer Ideen, zum Austausch in Netzwerken und zur kreativen Organisation von Protesten erweitert und geprägt. Aktuelle feministische Debatten werden in sozialen Netzwerken, Blogs, Vlogs (Videoblogs) und auch Podcasts angestoßen, aufgegriffen und weitergeführt. Im Sinne eines ,Netzfeminismus' eignen sich Frauen seit den 1990er Jahren Internet-Technologien und Online-Tools an und ermächtigen sich damit zur Bedeutungsproduktion und zur Partizipation an feministischen Diskursen (vgl. Stoltenhoff \& Raudonat, 2018, S. 132). Auch haben Online-Umgebungen neue Räume für die feministische Maxime „Das Private ist politisch“ (Pruchniewska, 2018, S. 811) geschaffen. Internetnutzer:innen haben die Möglichkeit, selbst zu Produzent:innen von Inhalten zu werden, ihre Geschichten zu erzählen und ihre eigene Identität innerhalb dieser Online-Räume zu konstituieren, sichtbar zu machen und auszuhandeln.

Während feministische Blogs und auch feministische Hashtags wie \#Aufschrei und \#MeToo bereits vielfach in wissenschaftlichen Studien thematisiert wurden, stellen feministische Podcasts ein bisher kaum erforschtes Phänomen dar (Hansen, 2013; Lenz, 2018; Sadowski, 2016; Tiffe \& Hoffmann, 2017). Im Feld der Gender Media Studies konzentrierten sich Wissenschaftler:innen bislang vor allem auf das feministische Potential von Podcasts für die Wissenschaftskommunikation und für pädagogische Kontexte, weniger auf unabhängige Podcasts und deren Macher:innen (Doane, McCormick \& Sorce, 2017; Fields, 2011; Gaden, 2009; Richardson \& Green, 2018). Die vorliegende explorative Studie will die Praktiken unabhängiger feministischer Podcaster:innen in den Blick nehmen und damit einen Beitrag zur Erweiterung des Forschungsfeldes leisten. Gerade unabhängiges Podcasting stellt eine spezifische Form der Online-Partizipation dar - mit einer eigenen subkulturellen Logik, die sich von institutioneller und traditioneller Radioproduktion abgrenzt (vgl. Millette, 2011, S. 2). In diesem Sinne wird feministisches Podcasting selbst zum netzfeministischen Akt und das Medium Podcast zu einem Sprachrohr für Menschen und Themen, die in Mainstream-Medien nicht gehört werden. In Anlehnung an Kellers Studie (Keller, 2013) zu feministischen Blogger:innen sollen feministische Podcasts als vernetzte Gegenöffentlichkeiten und der Aktivismus feministischer Podcaster:innen als kollektive und vernetzte feministische Praxis verstanden werden. Die Macher:innen feministischer Podcasts greifen feministische 
Perspektiven auf, ordnen aktuelle feministische Debatten ein und betrachten vielfältige Themen durch eine feministische ,Brille'. Mit dem vorliegenden Aufsatz soll ein Verständnis für die feministischen Identitäten, die Motive und Ansprüche sowie die Netzwerke feministischer Podcaster:innen entwickelt werden. Dafür werden zunächst theoretische Grundlagen sowie zentrale Aspekte des aktuellen Forschungsstands ausgeführt. Daraufhin werden der empirische Zugang und die Ergebnisse der Studie vorgestellt, gefolgt von einem abschließenden Fazit.

\section{Feministische Bewegungen und Identitäten}

Feminismus kann nicht als Überbegriff einer einheitlichen Idee oder Denkweise gelten, sondern umfasst vielmehr eine Vielzahl diverser und heterogener Feminismen, die sich im Laufe der Geschichte und in verschiedenen (thematischen und räumlichen) Kontexten herausgebildet und entwickelt haben (vgl. Drüeke, 2018, S. 20). Gemeinsam ist feministischen Denkweisen und Praktiken jedoch das Ziel einer strukturellen Veränderung von Machtverhältnissen bzw. die „Aufhebung von Unterdrückungs- und Marginalisierungsstrukturen und -mechanismen" (Thiessen, 2010, S. 41) in Bezug auf das Geschlecht. Im Kontext einer neoliberalen Gesellschaft, in der Diskurse von Autonomie und Individualität dominieren, ist die Formierung einer kollektiven feministischen Identität - und damit eines einheitlichen Verständnisses von Feminismus - schwieriger denn je (vgl. Fotopoulou, 2016, S. 3). Feministische Bewegungen sind heute geprägt von Postmoderne, Postkolonialismus und Poststrukturalismus sowie den Einflüssen eines globalen Kapitalismus und neuer Informations- und Kommunikationstechnologien (vgl. Dicker \& Piepmeier, 2003, S. 10). Viele Grundrechte und Freiheiten, für die frühere Feminist:innen gekämpft haben, erleben junge Frauen heute als selbstverständlich (ebd.). Sie haben frühere feministische Forderungen und Strategien umgedeutet und sich angeeignet sowie gleichzeitig neue Inhalte und Formen feministischen Aktivismus' entwickelt. Die Vielfalt heutiger Feminismen spiegelt sich in der Anerkennung der Diversität von Frauen-Identitäten wider, wobei das Geschlecht nicht mehr als übergeordnet identitätsstiftend wahrgenommen werden muss. Der frühere Anspruch einer kollektiven Frauen-Identität wurde erneuert und ersetzt durch ein poststrukturalistisches Verständnis fluider, vielschichtiger und facettenreicher Identitäten (vgl. Harris, o. J.a, S. 7). Gleichzeitig sind „Fragen von Ungleichheit und Differenz unter Frauen [...] in den Mittelpunkt des angloamerikanischen feministischen Diskurses gerückt" (Knapp, 2005, S. 2). Unter der Annahme, dass die unterschiedlichen Kategorien von Identitäten nicht voneinander trennbar sind, sondern sich überschneiden (engl. to intersect) und gegenseitig bedingen, wurde ,Intersektionalität' zu einem Paradigma feministischer Bewegungen (vgl. Walgenbach, 2012, S. 1). Mit dem Konzept der Intersektionalität werden die Wechselwirkungen von Geschlecht und weiteren sozialen Kategorien ${ }^{1}$

1 Aus der US-amerikanischen Tradition stammt die klassische Triade sozialer Kategorien von Race, Class and Gender. Diese Aufzählung wird häufig ergänzt um eine oder mehrere möglicher Differenzkategorien, wie Sexualität, Ethnizität, Kultur, Gesundheit, Alter, Religion, Nation usw. (vgl. Knapp, 2005, S. 2; 
bzw. Ungleichheiten erfasst (vgl. Lenz, 2010, S. 159; Piepmeier, 2009, S. 126; Walgenbach, 2012, S. 1).

Gegenläufig dazu werden strukturelle Ungleichheiten vermehrt als persönliche und individuell lösbare Probleme wahrgenommen (vgl. Baer, 2016, S. 20). Die für die Postmoderne charakteristische Tendenz der Individualisierung findet sich in heutigen feministischen Bewegungen wieder. Feministisches Handeln verschiebt sich von formalen Organisationsstrukturen auf die persönliche Ebene einzelner Akteur:innen, ,schwesterliche' Verbundenheit und Zusammenarbeit weichen eher lose geknüpften, solidarischen Netzwerken (vgl. Bock, 2010, S. 879; Harris, 2010, S. 478, o. J.a, S. 9). Im Kontext neoliberaler Diskurse gewinnen Konzepte wie Autonomie, Selbstverwirklichung und Unabhängigkeit an Bedeutung (Baer, 2016, S. 20). In diesem Sinne haben viele Feminist:innen einen spielerischen, an Popkultur orientierten Aktivismus entwickelt (vgl. Harris, 2010, S. 478; Keller, 2012, S. 440). Dabei wird immer wieder die Ambivalenz sichtbar zwischen dem Einsatz für feministische Ziele auf gesellschaftlicher Ebene durch Partizipation und Empowerment auf der einen Seite und einer individualistischen und teilweise profitorientierten Selbstvermarktung auf der anderen Seite (vgl. Pruchniewska, 2018, S. 3; Carstensen, 2019, S. 8).

\section{Feministische Gegenöffentlichkeiten im heutigen Netzfeminismus}

\subsection{Die Entwicklung feministischer Gegenöffentlichkeiten}

Für feministische Bewegungen waren und sind die Sichtbarkeit in und der Einfluss auf öffentliche Diskurse zentrale Anliegen. Dass Frauen der Zugang zu einer bürgerlichen Öffentlichkeit oft verwehrt blieb und auch heute noch bleibt, erhob die Deutung und Gestaltung von Öffentlichkeit „zu einer Schlüsselfrage der Emanzipationsbewegungen von Frauen" (Drüeke, 2019, S. 2) und zu einem „zentrale[n] Forschungsfeld der Geschlechterforschung“ (ebd.). Häufig wird in der wissenschaftlichen Auseinandersetzung mit feministischen Öffentlichkeiten Nancy Frasers Konzept der ,Gegenöffentlichkeiten' herangezogen (Clark-Parsons, 2017; Harris, o. J.b). Für Fraser (1992, S. 123) konkurriert die hegemoniale bürgerliche Öffentlichkeit mit „subaltern counterpublics“, in denen marginalisierte Gruppen Diskurse abseits hegemonialer Deutungshoheit führen und so die Deutungshoheit über ihre eigenen Identitäten und Anliegen erlangen. Fraser beschreibt das emanzipatorische Potential von Gegenöffentlichkeiten wie folgt:

"On the one hand, they function as spaces of withdrawal and regroupment; on the other hand, they also function as bases and training grounds for agitational activities directed toward wider

Lenz, 2010, S. 159). Wie Butler (1999, S. 182) anmerkt, steht immer „an embarrassed ,etc.' at the end of the list", da diese unter der Prämisse diskursiv konstituierter Identitäten niemals abgeschlossen sein könne. 
publics. It is precisely in the dialectic between these two functions that their emancipatory potential resides." (Fraser, 1992, S. 124)

Um sich selbst zu artikulieren, zu informieren und andere zu mobilisieren, erschaffen Frauen bereits seit 250 Jahren Gegenöffentlichkeiten, indem sie alternative Medien produzieren und verbreiten (vgl. Steiner, 1992, S. 121). Die Produktion feministischer Medien spielte von Beginn an eine zentrale Rolle für Frauenbewegungen - zunächst vor allem in Form von Printmedien, seit den 1960er und 1970er Jahren auch im Hörfunk (vgl. Gunnarsson Payne, 2012, S. 56; Steiner, 2012, S. 183). In Deutschland „bildete Louise Otto-Peters mit ihrer 1849 gegründeten Frauenzeitung den Anfang der organisierten Frauenbewegung" (Schmincke, 2019, S. 2). Seit den 1990er Jahren hat sich die Möglichkeit der Produktion alternativer Öffentlichkeiten noch einmal vervielfacht: „Die hegemoniale Struktur der Massenmedien wird im Internet aufgebrochen" (Engelmann et al., 2010, S. 76). Cyberfeminist:innen setzten in den 1990er Jahren große Hoffnungen auf das Potential des neuen ,Cyberspace'. Aufbauend auf der Vision einer Körper- und damit Geschlechtslosigkeit im Internet, sollten Frauen durch die Aneignung des Cyberspace neue Macht erlangen (vgl. Stoltenhoff \& Raudonat, 2018, S. 132; Drüeke, 2019, S. 3). Diese Utopie ist mittlerweile der Erkenntnis gewichen, dass Geschlechterverhältnisse in Technologien und Software eingeschrieben sind und sich patriarchale und diskriminierende Machtstrukturen im Netz fortschreiben, vielleicht sogar verstärken (vgl. Bücker, 2014, S. 118; Drüeke, 2018, S. 2). In diesem Spannungsfeld bewegen sich auch feministische Podcasts. Sie sollen in diesem Aufsatz als Gegenöffentlichkeiten charakterisiert werden, in denen ein diskursiver Raum für marginalisierte Stimmen geschaffen wird und ein emanzipatorisches Potential für feministische Diskurse entsteht. Damit stellen sie einen wichtigen Teil des heutigen Netzfeminismus dar.

\subsection{Der heutige Netzfeminismus}

Als Netzfeminismus wird zum einen eine „kritisch-feministische Perspektive auf netzpolitische Themen" (Aigner \& Schiff, 2019) verstanden, zum anderen umfasst er diverse Formen und Strategien feministischen Online-Aktivismus'. Im Allgemeinen bezeichnet Online-Aktivismus Aktivitäten und Kampagnen zivilgesellschaftlicher Akteur:innen, die das Internet nutzen, um zu informieren, zu organisieren und Bürger:innen für ihre politischen Ziele zu mobilisieren (vgl. Stier, 2017, S. 7, 27). Ebenso nutzen Netzfeminist:innen das Internet für vielfältige Möglichkeiten der Vernetzung, der Organisation und der Produktion von Inhalten (vgl. Daniels, 2009, S. 118). Ein wesentlicher Anteil dieser Aktivitäten passiert durch und innerhalb von sozialen Medien, die „als besonders user_innen-zentriert und partizipativ" (Carstensen, 2019, S. 2) gelten. Netzfeminismus muss nicht immer ergebnisorientiert oder auf massenmediale Diskurse ausgerichtet sein (vgl. Harris, o. J.b, S. 486). Gerade junge Feminist:innen erschaffen Gegenöffentlichkeiten im Netz „simply to exist as a space for expression and debate" (ebd.). Ihr Aktivismus findet häufig im Kleinen statt, in unkonventioneller und individueller Form. In diesem Sinne sollen 
auch feministische Podcaster:innen, unabhängig von ihrer Reichweite und ihrem gesellschaftlichen Einfluss, als Netzaktivist:innen verstanden werden.

In der Geschlechterforschung werden netzfeministischen Aktivitäten viele Potentiale zugeschrieben, da der Aktivismus im Netz mit vergleichsweise wenigen finanziellen und zeitlichen Ressourcen umgesetzt werden kann (vgl. Knappe \& Lang, 2014, S. 362). Feministische Akteur:innen werden online zu Produzent:innen eigener Inhalte. Vor allem in sozialen Medien kann eine Chance liegen für „subversive und widerständige Selbstpräsentationen, Empowerment und feministische Einflussnahme auf hegemoniale Öffentlichkeiten" (Carstensen, 2019, S. 9). Zudem können Artikulationsräume für diverse Identitäten und intersektionale Debatten entstehen, nicht nur öffentlich, sondern auch als , safe spaces' in geschlossenen Online-Communities (vgl. Daniels, 2009, S. 118; Fotopoulou, 2016, S. 4). Gleichzeitig bringt der Online-Aktivismus negative Aspekte mit sich, wie die Dynamik einer neoliberalen Selbstvermarktung und die Problematik unbezahlter Arbeit (vgl. Carstensen, 2019, S. 8f). Die kritische Nutzung sozialer Medien erfordert eine Auseinandersetzung mit Themen wie Überwachung, Datenschutz und der Abhängigkeit von profitorientierten Plattformen. Im besten Falle bedenken die Produzent:innen zudem die Zugänglichkeit und Exklusivität sowie die Unterschiede in Bezug auf die Nutzung von Internet-Technologien und Online-Angeboten (vgl. ebd.; Haidacher, 2014, S. 36). Letztlich sind digitale Öffentlichkeiten ,immer auch Schauplätze sozialer Kämpfe“ (Drüeke, 2019, S. 7). Im Netz können sich Geschlechterungleichheiten verschärfen sowie Gewalt und Diskriminierungen verstärkt werden.

Für Feminist:innen bedeutet die Sichtbarkeit durch Online-Aktivitäten gleichzeitig eine größere Angreifbarkeit. Antifeministische Auseinandersetzungen erreichen ein neues Ausmaß und werden „teilweise gewaltvoller zum Ausdruck gebracht als in herkömmlichen Öffentlichkeiten" (Drüeke, 2017, S. 144). In Bezug auf Podcasts stellt eine frühere Podcast-Studie aus Deutschland fest:

„Ein Blick in die Weblog-Foren selbst erfolgreicher deutschsprachiger Podcasts zeigt, dass inhaltliche Korrektur und Kommentierung durch Hörer und kritische, diskursive Auseinandersetzung unter diesen eher die Ausnahme als die Regel darstellt." (Mocigemba, 2007, S. 67).

Mocigemba (2007, S. 67) geht davon aus, dass im Vergleich zu Blogs politische Meinungsäußerungen in Kommentaren zu Podcasts gemäßigter ausfallen und „Diskurse weniger polemisch-konfrontativ ablaufen“. Als Erklärung nimmt er an, dass auditive Informationen in Podcasts mehr und vielfältigere soziale Hinweisreize liefern und damit antisoziales, ungehemmtes Verhalten reduzieren (ebd.). Es stellt sich die Frage, inwieweit sich diese Annahme auf feministische Podcasts übertragen lässt, die aufgrund ihrer Thematik bereits eine größere Anzahl abwertender Kommentare vermuten lassen. 


\subsection{Vernetzte Gegenöffentlichkeiten}

Durch den heutigen Netzfeminismus haben sich feministische Netzwerke verändert, ausgedehnt und diversifiziert (vgl. Keller, 2013, S. 154). OnlineNetzwerke spielen eine zentrale Rolle für aktuelle feministische Bewegungen und beeinflussen durch ihre strukturgebenden Eigenschaften feministische Gemeinschaften und Aktivitäten. Online-Netzwerke bieten vielfältige Möglichkeiten der Vernetzung von Gruppen und Einzelpersonen und werden beschrieben als temporär, fluide, interaktiv und unabhängig von Raum und Zeit (vgl. Garrison, 2000, S. 150; Keller, 2013, S. 144; Novoselova \& Jenson, 2018, S. 258). boyd (2011, S. 39) hat in diesem Zusammenhang den Begriff der ,networked publics' (dt. vernetzte Öffentlichkeiten) geprägt: „Networked publics are publics that are restructured by networked technologies". Für boyd (ebd.) beschreiben vernetzte Öffentlichkeiten einerseits den durch vernetzte Technologien entstehenden Raum, andererseits die durch die Nutzung dieser Technologien entstehenden virtuellen (diskursiven) Gemeinschaften. Vernetzte Öffentlichkeiten zeichnen sich durch vielfältige Interaktions- und Partizipationsmöglichkeiten aus, aber auch durch „eine Temporalität und Flüchtigkeit sowohl der Inhalte als auch der Akteur:innen" (Drüeke, 2019, S. 4). Das Konzept der vernetzten Öffentlichkeiten dient u.a. Studien zu feministischen Blogs als theoretische Grundlage (Keller, 2013; Novoselova \& Jenson, 2018). Keller (2013, S. 157) geht noch einen Schritt weiter und verknüpft danah boyds Konzept der , networked publics' mit Nancy Frasers Konzept der Gegenöffentlichkeiten: „the collectives of, girl feminists formed through blogging are best understood as what I'm calling ,networked counterpublics' ". Indem sie feministische Blogs als vernetzte Gegenöffentlichkeiten definiert, möchte Keller (ebd., S.146) neoliberale, individualistische und postfeministische Diskurse durchbrechen und den Aktivismus der Blogger:innen als kollektive und vernetzte feministische Praxis anerkennen. Gleichzeitig möchte sie die Netzwerke feministischer Blogger:innen von anderen OnlineNetzwerken abgrenzen und deren emanzipatorisches Potential im Sinne von Frasers , subaltern counterpublics' betonen (ebd., S. 160). In Anlehnung an Keller sollen auch feministische Podcasts in diesem Aufsatz als vernetzte Gegenöffentlichkeiten verstanden werden.

\section{Forschungsstand}

\subsection{Feministische Blogs: Studien mit feministischen Blogger:innen}

Feministische Blogs sind - im Gegensatz zu Podcasts - eine in der Geschlechterforschung viel beachtete Form des Netzfeminismus (vgl. Drüeke, 2017, S. 140). Da Blogs und Podcasts einige Parallelen aufweisen, soll der Forschungsstand zu feministischen Blogs im Folgenden angerissen werden, insbesondere in Bezug auf Ergebnisse aus qualitativen Interview-Studien mit feministischen Blogger:innen. 
Der Begriff Blog bzw. Weblog ist eine Zusammensetzung der Worte ,Web' und ,Logbuch' und beschreibt eine Website, auf der Einzelpersonen oder Autor:innen-Kollektive regelmäßig selbst geschriebene Beiträge veröffentlichen, wobei diese meist chronologisch geordnet sind beginnend mit dem aktuellsten Beitrag (vgl. Diemand, Mangold \& Weibel, 2007, S. 8; Washick, 2016, S. 113). Als ,feministische Blogosphäre' werden „kollaborative Zusammenschlüsse von Feminist:innen in und durch Blogs" (Drüeke, 2017, S. 140) beschrieben. Das Thema Vernetzung (untereinander, mit Leser:innen oder mit anderen Aktivist:innen) spielt für feministische Blogger:innen eine wesentliche Rolle. Beziehungsmanagement wird als charakteristischer Aspekt des Bloggens beschrieben (vgl. Schmidt, 2007, S. 52). Dieses findet einerseits innerhalb des Blogs durch Verlinkungen und Kommentare statt, andererseits sind Blogger:innen über den Blog hinaus häufig in sozialen Netzwerken aktiv (vgl. ebd.; Novoselova \& Jenson, 2018, S. 268). Novoselova/Jenson (ebd., S. 258) gehen zudem davon aus, dass der Austausch von Blogger:innen und ihrer Leser:innenschaft sich innerhalb der letzten fünf Jahre zu einem großen Teil vom Blog weg und in soziale Netzwerke, wie Facebook, Twitter und Instagram, verlagert hat. Für Keller (2013, S. 124) stellt „,community-building“ eine zentrale aktivistische Praxis feministischer Blogger:innen dar, zusammen mit „education" und „making feminism visible through performing feminist identities".

Feministische Bildung findet durch das Bereitstellen von Informationen sowie dem Einordnen von (aktuellen) Ereignissen und Themen in feministische Kontexte und Theorien statt und kann in Kommentaren fortgeführt werden (vgl. Novoselova \& Jenson, 2018, S. 265). Gleichzeitig tragen Blogger:innen durch die Produktion und das Veröffentlichen feministischen Wissens „zum Aufbau feministischer Online-Wissensarchive bei“ (Drüeke, 2017, S. 141).

Im Sinne von vernetzten Gegenöffentlichkeiten bilden Blogs Plattformen für den Austausch, die Aushandlung und die Sichtbarmachung feministischer Themen und Identitäten (vgl. Drüeke, 2017, S. 142; Keller, 2013, S. 83). Damit reproduzieren, hinterfragen und erweitern feministische Blogs marginalisierte Diskurse und haben das Potential, punktuell Einfluss auf hegemoniale bzw. massenmediale Diskurse zu nehmen (vgl. Shaw, 2012, S. 27). Die Sichtbarmachung marginalisierter Stimmen ermöglicht zudem die Sensibilisierung für und die Performance von intersektionalen Identitäten (vgl. Keller, 2013, S. 83). Gleichzeitig können Blogs einen geschützten Raum darstellen, in dem sich Feminist:innen untereinander artikulieren, ausprobieren und austauschen, ohne dass dabei zwingend das Ziel gesellschaftlicher Veränderungen im Vordergrund stehen muss (vgl. Harris, o. J.b, S. 489; Drüeke, 2019, S. 3). Bücker (2014, S. 117) beschreibt feministische Blogs in diesem Sinne als „emotionales Zuhause“, in dem Feminist:innen sich als Teil einer Gruppe ähnlich Denkender fühlen können.

Für Blogger:innen bedeutet die öffentliche Selbstdarstellung auch immer eine Verhandlung der eigenen Identität innerhalb der Diskurse und Konventionen der Blog-Gemeinschaft (vgl. Shaw, 2012, S. 228). In Interviews gaben einige 
feministische Blogger:innen an, der Prozess des Bloggens habe ihr Selbstverständnis, aber auch ihr Selbstbewusstsein als Feminist:innen verändert (vgl. Keller, 2013, S. 73f, 95). Für die Formung der eigenen feministischen Identität spielt neben dem Austausch innerhalb der Blog-Gemeinschaft auch die kontinuierliche Reflexion der eigenen feministischen Haltung und Praxis eine wichtige Rolle (vgl. Shaw, 2012, S. 228).

Einerseits bieten Blogs eine niedrigschwellige und unmittelbare Möglichkeit der öffentlichen Artikulation, unabhängig von Verlagen oder Medienunternehmen (vgl. Haidacher, 2014, S. 41). Andererseits darf nicht außer Acht gelassen werden, dass die Produktion eines Blogs nur für einen bestimmten Personenkreis realisierbar ist. Selbst wenn der Zugang zu einem Computer und schnellem Internet gegeben ist, bedeutet Bloggen einen großen Zeitaufwand, den nicht alle aufbringen können (vgl. Keller, 2013, S. 137). Geht es außerdem darum, sich eine bestimmte Reichweite und eine OnlineGemeinschaft aufzubauen, entscheiden bestehende Netzwerke und andere soziale Ressourcen darüber, wessen Blog gelesen, verlinkt und kommentiert wird. Die Unsichtbarkeit marginalisierter Gruppen wird somit auch online fortgeführt, denn , who gets to write, be read, and be followed on the Internet is shaped by hierarchies of inequality" (Novoselova \& Jenson, 2018, S. 261). Der mit dem Bloggen einhergehende Aufwand spiegelt sich auch darin wider, dass einige Blogger:innen das Führen eines Blogs als "work" oder "career" beschreiben (ebd., S. 263). Dazu gehört die Produktion des Blogs genauso wie dessen Bewerbung und die Beziehungspflege zur eigenen Leser:innenschaft (ebd.). Dieser Aufwand wird jedoch selten finanziell entlohnt.

\subsection{Unabhängige Podcaster:innen: ein Forschungsüberblick}

Das wissenschaftliche Interesse am Thema Podcasting hat in den letzten Jahren zugenommen und insbesondere im englischsprachigen Raum einige Forschungsschwerpunkte hervorgebracht. Hierzu gehören der Vergleich zum klassischen Hörfunk, pädagogische sowie ökonomische Potentiale, die Motivation von Hörer:innen und Produzent:innen, die Rolle von Podcasts in der gegenwärtigen Medien- und Kulturlandschaft sowie die Analyse einzelner Podcasts - insbesondere des True-Crime-Podcasts ,Serial' als Meilenstein des Podcast-Booms (vgl. Llinares, Fox \& Berry, 2018, S. 5ff). Für den vorliegenden Aufsatz ist insbesondere die Studienlage zur Motivation unabhängiger Podcaster:innen von Interesse. Zunächst sollen drei Studien aus dem englischsprachigen Raum kurz vorgestellt werden.

In einer qualitativen Studie führte Millette (2011, S. 3) u.a. Interviews mit fünfzehn unabhängigen Podcaster:innen in Montreal durch. Die Studienteilnehmer:innen beschreiben Podcasten als einen Teil ihrer Identität und ihren Podcast als Artikulationsraum für ihre eigenen Interessen - ein Raum, in dem die Authentizität und Subjektivität der Podcaster:innen im Vordergrund stehen (vgl. ebd., S. 8). Millette (ebd., S. 9) zeigt zudem auf, dass die Studienteilnehmer:innen das Podcasten mit der Nutzung von Blogs und sozialen Netzwerken 
verknüpfen, in denen sie ihren Podcast einbetten.

In einer explorativen Studie führte Markman (2012, S. 552) eine OnlineBefragung mit 147 englischsprachigen unabhängigen Podcaster:innen durch. Den typischen Studienteilnehmer beschreibt sie als älteren, hochgebildeten und berufstätigen Mann sowie als , unersättlichen' Social-Media-Nutzer (vgl. ebd. S. 553). Die meisten Podcasts der Befragten ermöglichten Hörer:innenFeedback in einem zugehörigen Blog oder per E-Mail (vgl. ebd., S. 554f.). Die Motivation unabhängiger Podcaster:innen untergliedert Markman (ebd., S. 555ff.) in sechs Kategorien: Interesse am Medium Podcast, Leidenschaft für ein Thema, Motivation durch Austausch und Feedback, persönliche Motive, Spaß am Produktionsprozess sowie finanzielle Interessen. In einer daran anschließenden Studie führten Markman \& Sawyer (2014, S. 26f)eine weitere Online-Befragung mit 120 unabhängigen Podcaster:innen durch, deren Teilnehmer:innen wiederum zu einem Großteil dem oben beschriebenen Typus entsprachen. Sie entwickelten aus den Antworten der Podcaster:innen vier Gründe, warum Menschen einen Podcasts starten: „public creativity, performance/promotion, long tail, and entertainment" (ebd., S. 27).

Im deutschsprachigen Raum sind Studien zu unabhängigen Podcaster:innen rar und liegen zudem schon einige Jahre zurück. Eine Ausnahme bildet eine 2019 durchgeführte umfassende Online-Befragung unter Podcaster:innen, die die Motivation und Persönlichkeit von Podcaster:innen sowie diesbezügliche Geschlechterunterschiede erfragt hat (Attig, 2019). Im Folgenden sollen diese sowie zwei Studien aus den Jahren 2006 und 2007 vorgestellt werden.

Mocigemba (2006, S. 6) erfragte in 15 qualitativen Interviews die Motivation unabhängiger Podcaster:innen - darunter 15 Männer und zwei Frauen. Sein Ziel war es, „eine Typologie von Sendemodi zu erstellen“, die aus sechs Typen besteht: der Explorer, der Personality Prototyper, der Journalist \& ThemenCaster, der Rebell, der Social Capitalist sowie der Social Gambler (ebd., S. 8ff.). Den Sendemodus des Explorer-Typs, der vordergründig die technischen Möglichkeiten erkunden will, bezeichnet Mocigemba (ebd., S. 11) als zentral für das Beginnen eines Podcasts. Zudem vermutet er, dass die zweitgrößte Gruppe deutschsprachiger Podcaster:innen dem Personality Prototyper zugeordnet werden kann und somit dem Motiv der Selbstdarstellung folgt (vgl. ebd., S. 14).

Eine weitere explorative Studie aus dem deutschsprachigen Raum stammt von Lauber, Wagner \& Theunert (2007), deren Fokus auf Jugendlichen und jungen Erwachsenen liegt. Die Ergebnisse basieren auf einer Online-Befragung sowie Gruppengesprächen mit je 14 Podcaster:innen (neun männlich und fünf weiblich) zwischen 13 und 26 Jahren (vgl. ebd., S. 27). Die zentralen Motive der Podcaster:innen „beziehen sich auf ihren Status als Early Adopters, auf ihr ,Sendungsbewusstsein' sowie darauf, dass sie Menschen erreichen wollen und ihnen die Rückmeldungen der Hörerinnen und Hörer wichtig sind" (ebd., S. 28). Gemeinsam ist den teilnehmenden Podcaster:innen die Leidenschaft und der Spaß am Podcasten, wobei ihnen inhaltliche Aspekte wichtiger seien als das technische Interesse (vgl. ebd., S, 30). 
In einer aktuellen Studie führte Attig (2019) eine Online-Befragung mit 653 Podcaster:innen durch [siehe auch Attig 2020 in dieser Sonderausgabe). Sie stellt fest, dass Frauen sowohl weniger Podcasts produzieren als Männer, als auch noch nicht so lange podcasten (vgl. ebd., S. 27). Für die meisten der befragten Podcaster:innen ist der Beginn und die Fortsetzung ihres Podcasts intrinsisch motiviert (vgl. Attig, 2019, S. 22). Als wichtigste Gründe für den Beginn des Podcasts nennt Attig (ebd., S. 20, 24) den Podcast als Medium, den Wunsch nach Wissensvermittlung, die Möglichkeit des Persönlichkeitsausdrucks und die Stärkung technischer Expertise. Zur Fortsetzung des Podcasts sind die Teilnehmenden zusätzlich durch die entstandene Community motiviert (vgl. ebd., S. 21). Der Podcast als Medium bleibt auch im Zeitverlauf der wichtigste Motivator (ebd.). Podcasterinnen heben sich in der Stichprobe durch eine geringere Technikaffinität von ihren männlichen Pendants ab (vgl. ebd., S. 30). Ihre anfängliche Motivation besteht eher darin, „ihrer Persönlichkeit und ihren Herzensthemen Ausdruck zu verleihen sowie aus dem Wunsch nach Monetarisierung" (ebd.). Noch mehr als die Podcaster setzen Podcasterinnen ihren Podcast aufgrund der Community, der eigenen Persönlichkeitsentwicklung und der Möglichkeit der Monetarisierung fort (ebd.).

Obwohl sich bisherige Studienergebnisse in manchen Punkten unterscheiden, werden wiederkehrende Motive unabhängiger Podcaster:innen deutlich, wie das Interesse am Medium Podcast und der Bedarf, sich einen Raum zur Artikulation bzw. zur Selbstdarstellung zu schaffen. Auch der Kontakt und Austausch mit Hörer:innen, Podcaster:innen und sonstigen Online-Netzwerken wird immer wieder betont.

\subsection{Frauen in deutschsprachigen Podcasts und der Podcast-Szene}

Frauenmedien sind nicht gleich feministische Medien. Dennoch kann Feminismus nicht ohne die Sichtbarkeit von Frauen und deren Belangen funktionieren - auch in der Podcastlandschaft. Diese war lange und ist auch heute noch zu einem großen Teil männerdominiert, jedoch nimmt die Zahl der von und mit Frauen produzierten Podcasts stetig zu (Markman, 2012; Reuter, o. J.; Tiffe \& Hoffmann, 2017). Medienforscherin Nele Heise schätzte deren Anteil 2018 im deutschsprachigen Raum auf etwa 15 Prozent (vgl. Bowman, o. J.). Genaue Zahlen oder Studien zur Gesamtheit aller deutschsprachigen Podcastenden gibt es bis heute keine. Zudem existiert kein einheitliches Podcast-Verzeichnis, in dem alle Podcasts aufgelistet wären. 2014 begann Heise unter dem Titel ,Frauenstimmen im Netz' eine Sammlung an Podcasts von und mit Frauen im deutschsprachigen Raum² zu kuratieren (vgl. Reuter, o. J.), auf der sich 2019 über 600 Podcasts befanden.

Ein Blick in die Blogosphäre zeigt: Obwohl der Frauenanteil unter Blog-

2 Die ursprüngliche Liste in Form eines Google-Sheets ist seit Juni 2019 offline. Ein Großteil der Podcasts von der Liste finden sich in der Podcast-Suchmaschine fyyd unter: https://fyyd.de/user/garneleh/ collection/frauenstimmen-im-netz (Zugriff am 06.09.2020). 
ger:innen überwiegt, werden die bekanntesten Blogs von Männern geschrieben (vgl. Hansen, 2013, S. 154). Hansen (ebd., S. 155) nennt als mögliche Gründe für dieses Ungleichgewicht Unterschiede in Bezug auf das Alter, die Inhalte und den Grad der Vernetzung. Auch in der Podcast-Szene gewinnt dieses Thema derzeit an Aufmerksamkeit. Seit Ende 2018 macht es sich die Plattform podcasterinnen.org zum Ziel, der Männerdominanz in der Podcast-Szene entgegenzutreten und „Podcasterinnen sowie nicht-binäre Podcastende und ihre Formate sichtbarer zu machen" "3. Auf der Website können Podcaster:innen ein Profil anlegen, um sich selbst und ihre Podcasts zu präsentieren. So sollen sie nicht nur von interessierten Hörer:innen entdeckt werden, sondern auch als Gäst:innen und Expert:innen für andere Podcasts, Workshops, Talks oder Panels auffindbar sein. Bowman (o. J.) stellt in einem Blog-Beitrag fest: „Trotz allem sind Frauen bei Branchentreffen wie der Podcast-Konferenz Subscribe eindeutig in der Unterzahl und auch bei Podcast-Panels oder in entsprechenden Medienberichten sind sie eher selten vertreten". Susanne Klingner schreibt im Rahmen eines von ihr und Katrin Rönicke moderierten Panels ${ }^{4}$ auf der Subscribe 2019 zur Diversität in der deutschen Podcastlandschaft:

„Jede und jeder kann Podcasts machen, und doch sind es vor allem Männer, weiße Männer, die das Podcast-Angebot dominieren. So ist das Angebot in Deutschland trotz des aktuellen Podcast-Booms recht öde: Dude-Podcasts, Ratgeber, Wellness, Finanz- und Marketingpodcasts. Der Hype 2018 hat nicht nur sehr viele neue Podcasts hervorgebracht, sondern vor allem sehr viel vom immer gleichen." (Klingner, o. J.)

\section{Empirischer Zugang}

Der empirische Zugang zum Forschungsfeld feministischer Podcasts erfolgt in der vorliegenden Arbeit durch das methodologische Verständnis der Grounded Theory. Die explorative Herangehensweise und das mit der Grounded Theory einhergehende bewusste und reflektierte Forschen hat mehrere Vorteile: Die Auseinandersetzung mit feministischen Podcasts ist in den Gender Media Studies noch sehr unterrepräsentiert, daher sind Offenheit und Flexibilität in diesem Forschungsfeld von ausgesprochener Relevanz. Wie bisherige Studien gezeigt haben, eignet sich die Grounded Theory zudem insbesondere dazu, die Aneignung des Mediums und die Bedeutungsproduktion durch die Podcaster:innen herauszuarbeiten und zu erklären (Gynnild, 2016; Lünenborg \& Meier, 2018; Shaw, 2012). Der durch den Kreislaufcharakter der Grounded Theory vergleichsweise hohe Aufwand „lohnt sich vor allem dann, wenn es um ein tieferes Verständnis des Medienhandelns geht" (Müller, 2018, S. 165). Mit der für die Forschungspraxis adaptierten Anwendung dieses Forschungsstils soll ein offenes und gegenstandsbezogenes Verstehen der Denkweisen und Praktiken

3 https://podcasterinnen.org/about (Zugriff am 04.10.2020).

4 Susanne Klingner, Katrin Rönicke und Barbara Streidl hosten u.a. den feministischen Lila Podcast. Das Panel mit dem Titel ,Zwei Männer unterhalten sich' kann auf YouTube angesehen werden unter: https: //www.youtube.com/watch?v=p5GgXH36jbY (Zugriff am 06.09.2020). 
unabhängiger feministischer Podcaster:innen ermöglicht werden. Zunächst soll ein Verständnis für die feministischen Identitäten der Podcaster:innen gewonnen werden - unter der Prämisse, dass deren (Selbst-)Verständnis von Feminismus variiert und veränderlich ist. In einer Interview-Studie mit Macher:innen feministischer Zines ${ }^{5}$ stellt Kempson (2015), 461 fest, dass unter den Befragten keine Einigkeit darüber herrscht, was ihre feministische Identität ausmacht. Keller schreibt in ihrer Dissertation über junge feministische Blogger:innen:

„Es gibt zahlreiche Gründe für dieses unterschiedliche Verständnis von Feminismus - Rasse, Klasse, Sexualität und andere Identitäten, häusliche Umgebung und sozialer Standort, bestimmte Interessen sowie aktivistisches Engagement und Bildung - um nur einige zu nennen." (Keller, 2013, S. 67)

Sie stellt zudem fest, dass der Prozess des Bloggens dazu geführt hat, dass sich die feministischen Identitäten der Blogger:innen mehrfach verändert haben (vgl. ebd., S. 73f.). Da in der vorliegenden Studie die Praktiken von Podcaster:innen im Vordergrund stehen, sollen diese auch in Bezug auf ihre feministischen Identitäten ergründet werden.

Die erste Forschungsfrage lautet dementsprechend:

FF1: Durch welche zentralen Praktiken konstituieren unabhängige feministische Podcaster:innen ihre feministischen Identitäten?

In einem zweiten Schritt sollen Motive und Ansprüche in den Fokus genommen werden. Podcasting ermöglicht auch technisch weniger erfahrenen Internetnutzer:innen das Erstellen und Veröffentlichen eigener Audio-Inhalte und doch sind feministische Podcasts bisher weit weniger verbreitet als feministische Blogs. Warum nutzen die Podcaster:innen dieses Medium? Welche Ansprüche stellen sie an die Umsetzung ihres Podcasts? Und lassen sich $\mathrm{Zu}$ sammenhänge zu ihren feministischen Praktiken herstellen? Bisherige Studien haben gezeigt, dass die am häufigsten genannte Motivation, einen eigenen Podcast zu betreiben, bei den (in der Mehrzahl männlichen) Podcaster:innen in der Begeisterung für das Medium liegt sowie in dem Wunsch nach Persönlichkeitsausdruck/Selbstdarstellung, Wissensvermittlung und der Erkundung der technischen Möglichkeiten (Attig, 2019; Markman, 2012; Mocigemba, 2006). Im Vergleich dazu zeigen Studien zu feministischen Blogs und Zines, dass dort folgende Motive im Vordergrund stehen: die Bildung einer Gemeinschaft, die Verbreitung von Informationen, das Sichtbarmachen von Feminismus sowie das Ausdrücken der eigenen Erfahrungen und Meinungen (Keller, 2013; Piepmeier, 2009). Aus diesen Erkenntnissen leitet sich die zweite Forschungsfrage ab:

FF2: Welche Motive und Ansprüche prägen die Podcasting-Praxis feministischer Podcaster:innen?

5 Als Zines werden unabhängige, nicht kommerzielle Publikationen bezeichnet, die über subkulturelle Netzwerke verbreitet werden (vgl. Kempson, 2015, S. 459). 
Mit dem Verständnis von feministischen Podcasts als vernetzte Gegenöffentlichkeiten sollen zuletzt die durch das Podcasten entstandenen Netzwerke im Fokus der Untersuchung stehen. Ein Blick auf die Webpräsenz feministischer Podcaster:innen zeigt, dass diese nicht nur eine Website für ihren Podcast pflegen, sondern ebenfalls in sozialen Netzwerken präsent und aktiv sind. In Bezug auf feministische Blogs beschreibt Keller (vgl. ebd., S. 124) das Bilden von Gemeinschaften als zentrale feministische Praxis. Abschließend soll daher ergründet werden, inwiefern sich die Podcaster:innen z.B. mit Hörer:innen, Gäst:innen und anderen Podcaster:innen vernetzen und ob der Austausch innerhalb dieser Netzwerke für sie eine Rolle spielt. Diese Überlegungen resultieren in der dritten und letzten Forschungsfrage:

FF3: Inwiefern entstehen durch das Podcasten Netzwerke und wie agieren unabhängige feministische Podcaster:innen innerhalb dieser Netzwerke?

Für die vorliegende Studie wurden Interviews mit sechs unabhängigen feministischen Podcasterinnen geführt. Die Interviews wurden innerhalb von drei Wochen im März 2019 telefonisch bzw. online per Skype und Google Hangouts durchgeführt und dauerten je etwa eine halbe bis dreiviertel Stunde. Für die Interviews wurden Podcasterinnen ausgewählt, die ihren Podcast explizit als feministisch oder Feminismus als Kernthema ihres Podcasts bezeichnen. Für die Grounded Theory ist es zentral, einerseits möglichst homogene (minimale Kontrastierung), andererseits möglichst heterogene (maximale Kontrastierung) Fälle miteinander zu vergleichen (vgl. Strübing, 2018, S. 41). Die Relevanz der Vergleiche und Kontrastierungen zieht sich durch den gesamten Forschungsprozess, beginnend mit der Fallauswahl (vgl. ebd.). In der vorliegenden Stichprobe finden sich Unterschiede und Ähnlichkeiten in Bezug auf das Format, den Entstehungszeitpunkt, die Reichweite und die räumliche Verortung (Deutschland oder Österreich). Einen Überblick über die Podcasts der Stichprobe bietet Tab. 1, in der die Podcast-Macherinnen, das Entstehungsjahr und das Format der Podcasts aufgelistet sind.

\begin{tabular}{|c|c|c|c|c|}
\hline Podcast & Macherin(nen) & Seit & Format & Website \\
\hline Lila Podcast & $\begin{array}{l}\text { Katrin Rönicke, Susanne } \\
\text { Klingner, Barbara Streidl }\end{array}$ & 2013 & GesprächsPodcast & lila-podcast.de \\
\hline Feuer und Brot & $\begin{array}{l}\text { Maximiliane Häcke, } \\
\text { Alice Hasters }\end{array}$ & 2016 & GesprächsPodcast & feuerundbrot.de \\
\hline Große Töchter & Beatrice Frasl & 2018 & $\begin{array}{l}\text { Gesprächs- und } \\
\text { Solo-Podcast }\end{array}$ & grossetoechter. podbean.com \\
\hline Jeannes Heldinnen & Jeanne Drach & 2017 & Interview- Podcast & ohwow.eu/jeannes- heldinnen \\
\hline 100 Frauen & Miriam Steckl & 2019 & Interview- Podcast & 10ofrauenpodcast. podigee.io \\
\hline Reichlich Randale & Rebecca Görmann & 2017 & $\begin{array}{l}\text { Gesprächs- und } \\
\text { Solo-Podcast }\end{array}$ & reichlich- randale.de \\
\hline
\end{tabular}

Der Lila Podcast ${ }^{6}$ ist einer der bekanntesten und am längsten aktiven feministischen Podcasts in Deutschland. Die Macherinnen Susanne Klingner, Katrin

6 https://lila-podcast.de/ (Zugriff am 04.10.2020). 
Rönicke und Barbara Streidl sprechen seit 2013 wöchentlich über aktuelle feministische Themen und Debatten, meistens mit einer oder mehreren Gäst:innen. Das Interview für die vorliegende Studie wurde mit Katrin Rönicke geführt. In dem seit 2016 aktiven Podcast Feuer und Brot ${ }^{7}$ sprechen die beiden Freundinnen Maximiliane Häcke und Alice Hasters einmal im Monat über feministische Themen und ihre persönlichen Erfahrungen. Meistens podcasten die beiden zu zweit, manchmal mit Gäst:innen. Im Interview sprach die Autorin dieser Studie mit Maximiliane Häcke. Neben den deutschen sind auch zwei österreichische Podcasts Teil der Stichprobe: Große Töchter $^{8}$ und Jeannes Heldinnen ${ }^{9}$. Beatrice Frasl startete 2017 bereits ihren ersten (englischsprachigen) feministischen Podcast She Who Persisted ${ }^{10} .2018$ kam mit Große Töchter ein feministischer Podcast speziell für Österreich dazu, in dem sie sich zumeist mit Gäst:innen unterhält, manchmal allein zu bestimmten Themen und Ereignissen Stellung bezieht. Jeanne Drach führt in ihrem Podcast Jeannes Heldinnen Interviews mit inspirierenden Frauen, wobei die Gespräche von feministischen Themen geprägt sind. Auch Miriam Steckl führt in ihrem Podcast 100 Frauen $^{11}$ Interviews, in denen sie etwa alle zwei Wochen mit feministischen Aktivist:innen spricht. Rebecca Görmann hat seit 2017 mit Reichlich Randale ${ }^{12}$ einen unregelmäßig erscheinenden Personal Podcast (auch Tagebuch-Podcast genannt) mit feministischem Einschlag. Gleichzeitig podcastet sie in einigen anderen Formaten mit feministischer Perspektive, u.a. in den Podcasts Kulturpessimist:innen ${ }^{13}$, Filmlöwinnen ${ }^{14}$ und FRÜF - Frauen reden über Fußball ${ }^{15}$.

\section{Ergebnisse}

Die Ergebnisse der Studie sollen an dieser Stelle anhand von drei Kernkategorien dargestellt werden, die analog zu den drei vorangehenden Forschungsfragen herausgebildet wurden ${ }^{16}$ : erstens Feminismus als Reflexions- und Aushandlungsprozess, zweitens feministisches Podcasting als Ausdruck von $\mathrm{Zu}-$ gehörigkeit und Unabhängigkeit sowie drittens Austausch und Vernetzung als Kernpraxis feministischer Podcaster:innen. In Bezug auf feministische Praktiken hat sich gezeigt, dass die interviewten Podcasterinnen ein gemeinsames Verständnis von Feminismus als Reflexions- und Aushandlungsprozess haben, in dem die Relevanz eines intersektionalen Feminismus und die Auseinandersetzung mit marginalisierten Perspektiven eine zentrale Rolle spielen. Des Weiteren stellte sich feministisches Podcasting als eine Praxis heraus, die geprägt ist von dem Bewusstsein, dass der eigene Podcast eingebunden ist in

\footnotetext{
7 https://feuerundbrot.de/ (Zugriff am 04.10.2020).

8 http://grossetoechter.podbean.com/ (Zugriff am 04.10.2020).

9 https://www.ohwow.eu/jeannes-heldinnen (Zugriff am 04.10.2020).

10 http://shewhopersisted.com/ (Zugriff am 04.10.2020).

11 https://100frauenpodcast.podigee.io/ (Zugriff am 04.10.2020).

12 https://www.reichlich-randale.de/ (Zugriff am 04.10.2020).

13 https://www.kultpess.de/ (Zugriff am 04.10.2020).

14 https://filmloewin.de/podcast/ (Zugriff am 04.10.2020).

15 https://www.fruef.de/ (Zugriff am 04.10.2020).

16 Der Kodierprozess für die Datenauswertung orientierte sich an den von Strauss \& Corbin (1998), 30ff beschriebenen Schritten des offenen, axialen und selektiven Kodierens, welche nicht strikt nacheinander, sondern in einem zirkulären Prozess bearbeitet werden, bis sich eine Kernkategorie aus dem Datenmaterial heraus entwickelt.
} 
gesellschaftliche und persönliche Beziehungen und Zusammenhänge sowie in feministische Bewegungen und Ideen. Die Bezugnahme der Podcasterinnen auf andere Personen und Personengruppen ist dementsprechend fest in ihr verankert und steht nicht im Widerspruch zu dem Gefühl, ein ,eigenes Ding' zu machen und mit dem Podcast unabhängig von äußeren Zwängen zu agieren. Zuletzt stellen Vernetzung und insbesondere der Austausch mit Hörer:innen eine Kernpraxis der Podcasterinnen dar, die für sie einen Mehraufwand, aber auch einen Zugewinn bedeutet.

\subsection{Feminismus als Reflexions- und Aushandlungsprozess}

Die Interviews haben gezeigt, dass die Podcasterinnen ihre feministischen Identitäten immer wieder neu aushandeln und sich dieser Prozesshaftigkeit auch bewusst sind. Ihre feministischen Identitäten konstatieren sich in einem Feld aus sozialen Einflüssen, persönlichen Erfahrungen und der medialen Präsenz anderer Feminist:innen, insbesondere in sozialen Netzwerken. Selbstreflexion und die konstante Auseinandersetzung mit der eigenen Meinung spielen für die Interviewten eine zentrale Rolle. Sie sind zwar auf unterschiedlichen Wegen zum Feminismus gekommen und unterscheiden sich zu Beginn des Podcastens in Bezug auf ihr feministisches Vorwissen und ihre Erfahrung mit feministischem Aktivismus. Dennoch zeichnete sich in den Interviews ein einheitliches Verständnis ihrer feministischen Praxis als ständiger Reflexions- und Aushandlungsprozess ab. Eine der Podcasterinnen erklärt:

„Ich verändere meine Meinung auch recht oft und ich glaube, das ist auch wichtig, dass man seine Meinung verändert. Trotzdem ist es so, dass es klar ist, [...] für mich ist Feminismus ein Grundstein für meine Vision einer neuen Gesellschaft." (J. Drach, Interview, 11. März 2019)

Ergänzend hierzu bedeutet Feminismus für die Podcasterinnen in einem weiteren Schritt, aktiv zu werden und Stellung zu beziehen. Dies scheint insofern besonders relevant, da die Produktion eines feministischen Podcasts ebenfalls bedeutet, aktiv zu werden und den eigenen feministischen Standpunkt zu veröffentlichen.

Im Gegensatz zu der Annahme eines individualisierten Feminismus, fühlen sich viele der Podcasterinnen als Teil einer feministischen Bewegung oder zumindest als Teil einer in Deutschland/Österreich aktiven Gruppe von Feminist:innen. Gleichzeitig betonen manche der Interviewten eine Distanzierung zu früheren feministischen Bewegungen, insbesondere zu Alice Schwarzer. Selbst haben die Interviewten ebenfalls die Erfahrung gemacht, sich immer wieder für ihren Feminismus rechtfertigen zu müssen, auch vor anderen Feminist:innen. Dass in diesem Zusammenhang häufig ein Mangel an Solidarität unter Feminist:innen und unter Frauen im Allgemeinen proklamiert wurde, scheint einer kollektiven feministischen Bewegung wiederum entgegenzustehen. Eine der Podcasterinnen stellt fest: „Dieses gegenseitige Abwerten und Bewerten und Herunterspielen von anderen Frauen, das ist 
auch das, was immer noch, glaube ich, die größte Hürde ist in der Solidarität" (M. Häcke, Interview, 11. März 2019).

Neben dem Wunsch nach mehr Solidarität unter Feminist:innen wurde von den Interviewten das Bedürfnis nach einer feministischen Streitkultur geäußert. Beides muss sich nicht ausschließen, doch in der Praxis scheint oft Uneinigkeit zu herrschen, wie konstruktiv gestritten und gleichzeitig solidarisch zueinander gehalten werden kann.

Obwohl das Thema Intersektionalität nicht im Interview-Leitfaden auftaucht, wurde dessen Relevanz immer wieder von den Podcasterinnen betont. Das Konzept der Intersektionalität scheint somit nicht nur in der Geschlechterforschung in Deutschland angekommen zu sein, sondern ist auch fest in den Feminismus dieser aktiven Netzfeministinnen eingeschrieben. In Einklang damit steht die Forderung der Podcasterinnen, marginalisierte Perspektiven in der Öffentlichkeit mehr wahrzunehmen und anzunehmen:

„Wenn wir losgehen und z.B. muslimischen Frauen irgendwie absprechen, dass sie über sich selber sprechen können und dass sie ihre eigenen Belange selber formulieren dürfen und dass nicht über ihren Kopf hinweg gerichtet und entschieden wird, dann sieht man einfach, dass es mit dem Feminismus da nicht so weit her ist." (M. Häcke, Interview, 11. März 2019)

Im Laufe der Studie zeigte sich, dass sich die feministischen Praktiken der Interviewten auch in deren Podcasting-Praxis wiederfinden und deren Motive und Ansprüche in Bezug auf den Podcast prägen. Umgekehrt wurde ebenso deutlich, dass durch den Podcast der feministische Reflexions- und Aushandlungsprozess der Interviewten weiter angestoßen wird und dass Zuhören und Lernen für die Interviewten auch beim Podcasten eine zentrale Rolle spielen.

\subsection{Feministisches Podcasting als Ausdruck von Zugehörigkeit und Unabhängigkeit}

Als zentrales Ergebnis der Studie hob sich feministisches Podcasting als eine Praxis hervor, die geprägt ist von der Zugehörigkeit zu einer gemeinsamen Bewegung, Idee oder Community und damit auch von dem Wunsch, die Meinungen, Perspektiven, Ansprüche und Möglichkeiten anderer Personen und Personengruppen mitzudenken und einzubeziehen. Diese Erkenntnis fand sich sowohl in der Motivation der Podcasterinnen wieder, als auch in den Ansprüchen bei der Umsetzung des Podcasts und bei den späteren Entwicklungen und Erfahrungen. Gleichzeitig dient Podcasting den Interviewten als ein Medium, das ihnen viele Freiheiten lässt und in dem sie unabhängig von vielen externen Faktoren agieren können, insbesondere unabhängig von etablierten Medienunternehmen und ökonomischen Zwängen.

In Bezug auf die Motivation der Interviewten zeigten sich die Begeisterung für das Medium Podcast und der Wunsch, einen Artikulationsraum für sich und andere zu schaffen, als zentrale Kategorien. Das Interesse am 
Medium Podcast beschreiben frühere Studien mit unabhängigen Podcaster:innen ebenfalls als wichtiges Motiv (Attig, 2019; Markman, 2012). Bei den interviewten Podcasterinnen lag dieses Interesse jedoch weniger in der Ergründung technischer Möglichkeiten, sondern war verbunden mit den Vorteilen gesprochener Sprache sowie sozialen Implikationen des Mediums durch die leichtere Zugänglichkeit für Hörer:innen. Gleichzeitig scheinen die Eigenschaften und damit verbundenen Vorteile des Mediums bei den Podcasterinnen mehr im Vordergrund zu stehen als in vergleichbaren Studien mit feministischen Blogger:innen (Keller, 2013; Novoselova \& Jenson, 2018). Durch die gesprochene Sprache seien Podcasts „einfacher zugänglich auch für andere Zielgruppen, die sich vielleicht nicht hinsetzen und irgendwie einen zehnseitigen Artikel lesen" (B. Frasl, Interview, 25. März 2019). Die Zugänglichkeit für Hörer:innen wollen die Interviewten auch später im Laufe des Podcastens bewahren. Sie zeigten ein Bewusstsein dafür, dass das Thema Feminismus häufig auf Unverständnis oder Ablehnung stößt und wollen gerade deshalb möglichst viele Menschen erreichen und komplexe Inhalte verständlich und undogmatisch vermitteln:

„Insofern wäre mein Ziel einfach echt, immer wach und offen zu sein [...] und gleichzeitig niemals sozusagen diese Brücke, die wir glaube ich schon geschaffen haben zu den Leuten, die gerade erst anfangen, die nicht zu verlieren, sondern immer diese Brücke zu behalten, über die jeder und jede gehen kann, jederzeit." (K. Rönicke, Interview, 11. März 2019)

Gleichzeitig steht diese gewünschte Zugänglichkeit für Menschen ohne Vorwissen dem Gefühl mancher Podcasterinnen gegenüber, sich mit ihrem Podcast in einer ,Blase' zu befinden oder nur in feministischen Kreisen gehört zu werden.

Als ein weiteres zentrales Motiv für das Starten einen Podcasts zeichnete sich der Wunsch ab, einen Artikulationsraum für sich und andere zu schaffen:

„Also ich muss mir einen Raum schaffen zur Artikulation, auch gemeinsam mit anderen, und ich muss irgendwo hin mit dieser Wut. [...] Ich hatte das Gefühl, ich muss jetzt irgendwie so feministische Gegenöffentlichkeiten schaffen." (B. Frasl, Interview, 25. März 2019)

Der Wunsch nach einem Artikulationsraum wird in früheren Studien als wichtiges Motiv unabhängiger Podcaster:innen genannt, wobei dieses sich überwiegend auf den Ausdruck der eigenen Persönlichkeit bezieht (Attig, 2019; Millette, 2011; Mocigemba, 2006). Bei den interviewten Podcasterinnen hat sich gezeigt, dass diese einen Artikulationsraum nicht nur zur Darstellung der eigenen Meinungen und Erfahrungen schaffen, sondern dass sie auch andere Perspektiven berücksichtigen und einbeziehen wollen. Mehrfach hoben sie die Relevanz der Sichtbarmachung diverser, marginalisierter und intersektionaler Perspektiven hervor. Diese Forderung findet sich bereits in der Auseinandersetzung der Podcasterinnen mit dem Thema Intersektionalität und einem Verständnis für die Vielfalt von Frauen-Identitäten. Intersektiona- 
lität wurde von den meisten Interviewten als mehr oder weniger neuer Aspekt, aber dennoch als sehr präsent und wichtig für ihren Feminismus beschrieben. Zwar spielen bei der Wahl von Themen und Gäst:innen auch persönliche Interessen eine entscheidende Rolle, diese sind jedoch immer beeinflusst von dem Wunsch, bei der Gestaltung des Podcasts diversen Stimmen einen Raum zu geben. Eine der Podcasterinnen beschreibt ihre Rolle wie folgt:

„Ich glaube, ich habe sehr große Wirkung dadurch, wen ich einlade und was ich damit sage. Das war mir am Anfang auch nicht so bewusst. [...] Ich fühle mich so ein bisschen, wie eine Taschenlampe und es ist jetzt meine Verantwortung, dort wo ich hinleuchte, dort werden auch die Leute gesehen." (M. Steckl, Interview, 18. März 2019)

Weitere Gründe, einen Podcast zu starten - und vor allem einen feministischen Podcast - waren für die Interviewten erstens eine Leidenschaft für das Thema Feminismus bzw. ein Bezug zu dem Thema aus einer persönlichen Betroffenheit heraus, zweitens der Wunsch nach gesellschaftlichen Veränderungen und drittens ein Bedarf an weiblichen Vorbildern. Frühere Studien haben gezeigt, dass viele unabhängige Podcaster:innen eine Leidenschaft für ein bestimmtes Thema mitbringen und diese sie zum Podcasten motiviert (Markman, 2012; Millette, 2011). Die Interviewten beschreiben ihren Feminismus einerseits als Leidenschaft, andererseits als ein häufig durch persönliche Betroffenheit und Erfahrungen aus dem eigenen Alltag emotional besetztes Thema.

In ihren Podcasts beschäftigen sich die Interviewten nicht nur mit dem Thema Feminismus, sondern haben teilweise auch das Ziel, gesellschaftliche Veränderungen anzustoßen, um letztlich strukturelle Machtverhältnisse aufzubrechen. Zwar sehen sie ihren Einfluss auf hegemoniale Diskurse kritisch, doch auf individueller Ebene haben sie durchaus die Erwartung, Veränderungen zu bewirken sowie neue Perspektiven zu eröffnen. Diesen Einfluss bekommen sie durch das Feedback von Hörer:innen und Gäst:innen widergespiegelt. Auch in der Podcastlandschaft sehen die Interviewten Möglichkeiten des Einflusses, indem sie als Vorbilder für neue Podcast-Macher:innen dienen und damit zu einer größeren Bandbreite feministischer und diverser Podcasts beitragen: „Ich glaube, dass gerade durch die Repräsentation von u.a. uns, aber auch noch vielen anderen, der Mut wächst, da auch selber [...] etwas zu gestalten und mitgestalten zu wollen" (M. Häcke, Interview, 11. März 2019).

Neben dem eigenen Einfluss gehören der eigene Lernprozess und der Umgang mit Kritik zu den Erfahrungen, die die Interviewten mit dem Podcasten gemacht haben. Dass die Podcasterinnen ihre feministische Identität als einen Reflexions- und Aushandlungsprozess verstehen, spiegelt sich darin wider, dass Podcasten für viele der Interviewten ein kontinuierliches Lernen und Hinterfragen bedeutet. Dabei wird Lernen von den meisten der interviewten Podcasterinnen als begleitender Effekt des Podcastens beschrieben. Für zwei der Interviewten war der Wunsch zu lernen bereits ein Motiv für den Start ihres Podcasts - in Zusammenhang mit einem Bedarf an weiblichen Vorbildern. Der Lernprozess wird durch die intensive Beschäftigung mit Themen für eine Folge sowie durch den Austausch mit Gäst:innen und Hörer:innen angestoßen. 
Er bedeutet für die Podcasterinnen insbesondere eine kritische Auseinandersetzung mit eigenen Denkmustern und Begrifflichkeiten im eigenen Sprachgebrauch. Dies zeigt sich auch in einem wertschätzenden Umgang mit konstruktiver Kritik von Hörer:innen, die sich die Interviewten zu Herzen nehmen. Negative Erfahrungen machten manche der Interviewten mit Kritik an ihrem Podcast aus feministischen Kreisen.

Des Weiteren wird die Praxis der Podcasterinnen von dem Medium als solches beeinflusst. An dieser Stelle soll daher abschließend auf den Eindruck der Interviewten von Podcasting als einfaches und schnell zugängliches Medium eingegangen werden. Ihren eigenen Zugang zum Podcastmachen beschreiben die Interviewten als ungeplantes Ausprobieren und bestätigen die Niedrigschwelligkeit, die dem Medium nachgesagt wird. Gleichzeitig ermöglicht diese Niedrigschwelligkeit es ihnen, unabhängig von Medienunternehmen und Redaktionen zu agieren und mit dem Podcast ihr ,eigenes Ding' zu machen, eigene Ideen umzusetzen und eigene Interessen einzubringen - ein Aspekt, der für die Podcasterinnen nicht in Konflikt steht mit dem Anspruch, Rücksicht auf diverse Perspektiven und Meinungen zu nehmen. Die Aspekte Niedrigschwelligkeit und Unabhängigkeit spielen für die Wahrnehmung von Podcasting als demokratisches Medium eine zentrale Rolle, da potentiell jede:r eigene Audio-Inhalte produzieren und veröffentlichen kann. Insbesondere Smartphones, welche auch einige der interviewten Podcasterinnen zunächst nutzten, machen es durch technische Neuerungen (verbesserte Mikrofone, Podcast-Aufnahme-Apps) heute leichter denn je, einen eigenen Podcast zu kreieren. Warum es dennoch wenige feministische Podcasts gibt oder viele schnell wieder eingestellt werden, kann an dem überraschend hohen Arbeitsaufwand liegen, den viele der Podcasterinnen beschreiben und zudem anfangs unterschätzt haben:

„Bei mir dauert es schon um die acht Stunden, bis ein Podcast dann hinterher geschnitten ist. Ich hatte mir eigentlich vorgenommen, wöchentlich $\mathrm{zu}$ machen, habe aber jetzt auch einen Vollzeitjob angenommen und komme überhaupt nicht hinterher." (M. Steckl, Interview, 18. März 2019)

Selbst mit technischen Vorkenntnissen ist die Podcast-Produktion zeitintensiv und damit nur für solche Feminist:innen zugänglich, die diese weitestgehend unbezahlte Arbeit in ihrer Freizeit leisten können. Die Finanzierung durch Werbung lohnt sich erst ab einer gewissen Reichweite, wobei manche der Podcasterinnen diese Option bereits von Anfang an ausschließen. Zwei der Interviewten, Katrin Rönicke und Jeanne Drach, sind Mitgründerinnen eigener Podcast-Labels (hauseins und $\mathrm{OH}$ WOW) und kommen damit dem mehrfach geäußerten Wunsch näher, Podcasting als Job und nicht nur als Hobby zu betreiben:

„Die absolute Traumvorstellung [ist] natürlich, dass es irgendwann mal etwas ist, wovon man leben kann. Weil ich liebe es zu podcasten und es wäre einfach voll cool, wenn ich sozusagen nicht nebenbei fünf Jobs hätte, sondern mich einfach wirklich aufs Podcasten kon- 
zentriere. Also ich wäre gerne hauptberufliche Podcasterin. Das wäre so mein Hauptziel. Das ist im Moment noch sehr utopisch." (B. Frasl, Interview, 25. März 2019)

\subsection{Austausch und Vernetzung als Kernpraxis feministischer Podcaster:innen}

Zuletzt soll im Folgenden auf die Vernetzung durch das Podcasten eingegangen werden und insbesondere auf den Austausch mit Hörer:innen als Kernpraxis der feministischen Podcasterinnen. In den Interviews wurden unterschiedliche Schwerpunkte, Motive und Umgangsweisen mit Online-, aber auch Offline-Netzwerken deutlich. Es hat sich gezeigt, dass die Präsenz in sozialen Netzwerken für die Interviewten zum Podcasten dazu gehört. Auch wurde ersichtlich, dass der größtenteils online stattfindende Austausch und die Vernetzung zentrale Aspekte ihrer Aktivitäten als Podcasterinnen darstellen. Dabei haben sich insbesondere Instagram und Twitter als relevante Plattformen abgezeichnet, wobei zumindest von einigen der Podcasterinnen erstere eher für den Kontakt zu Hörer:innen und letztere eher für die Vernetzung mit Journalist:innen, Feminist:innen und Podcaster:innen genutzt wird.

Die Interviewten sind unterschiedlich stark in feministischen Kreisen und Podcasting-Communities vernetzt, teilweise bereits vor und teilweise erst mit dem Podcasten. So variiert auch die persönliche Bedeutung des Podcastens für den Aufbau dieser Netzwerke stark und manifestiert sich zudem auf unterschiedlichen Ebenen - sei es im Sinne freundschaftlicher oder eher in Bezug auf thematische/professionelle Kontakte. Es entsteht der Eindruck, dass bestimmte Personenkreise in Online- und Offline-Netzwerken unter sich bleiben, auch in Bezug auf eine eher geringe Vermischung zwischen Personen aus Österreich und Deutschland. Während dieser Netzwerk-Aspekt unterschiedliche Relevanz für die Interviewten hat, stellte sich der Austausch und aktive Umgang mit der Hörer:innenschaft als Kernpraxis der interviewten Podcasterinnen heraus. So erzählt Katrin Rönicke, „dass die Interaktion mit denen, die uns hören, sehr lebhaft ist" (Interview, 11. März 2019) und auch Maximiliane Häcke gibt an, dass sie „rege mit Leuten schreibt und Nachrichten bekommt und hin und her schreibt" (Interview, 11. März 2019). Für die meisten Interviewten ist neben E-Mails Instagram der wichtigste Kanal für diesen Austausch.

Die Kommunikation mit Hörer:innen kann auf verschiedenen Ebenen von Bedeutung sein. Sei es als Bestärkung, Anregung oder Kritik, der zentrale Stellenwert der Hörer:innen-Community wird in allen Interviews deutlich. Dies entspricht den Erkenntnissen aus Studien mit feministischen Blogger:innen, die das Bilden von Gemeinschaften als zentrale feministische Praxis der Blogger:innen beschreiben (Keller, 2013; Novoselova \& Jenson, 2018). Wie Novoselova und Jenson (ebd., S, 258) bereits bei feministischen Blogs feststellen, verlagert sich auch der Austausch mit der Hörer:innenschaft der interviewten 
Podcasterinnen häufig in soziale Netzwerke. Die Abrufbarkeit von Podcasts über unterschiedlichste Plattformen macht es quasi unmöglich, mit einer Kommentarfunktion auf der podcasteigenen Website alle Hörer:innen zu erreichen. Gleichzeitig hat sich gezeigt, dass die Kommunikation häufig im Privaten - also über Privatnachrichten oder E-Mails - stattfindet und nicht als Kommentare in sozialen Netzwerken für alle einsehbar ist. So entstehen seltener öffentliche Diskussionen oder ein Austausch unter den Hörer:innen. Dies gilt jedoch nicht nur für feministische Podcasts, sondern für Podcasts im Allgemeinen (Mocigemba, 2007, S. 65f). Gleichzeitig bemühen sich die Interviewten, den Umgang mit ihrer Community weiterzuentwickeln, der regen Kommunikation mit den Hörer:innen gerecht zu werden und diese teilweise aktiv zu fördern. Neue Kommunikations-Tools eröffnen weitere Möglichkeiten, den Austausch anzuregen und die Hörer:innenschaft einzubeziehen. Durch das Beziehungsmanagement der Podcasterinnen können ihre Communities wachsen und sich umfassende Netze bilden, in denen Hörer:innen, aber auch andere Podcaster:innen, untereinander vernetzt sind und gemeinsam an Stärke und Zusammenhalt gewinnen. Das Wir-Gefühl innerhalb einer Podcast-Community kann so den eigenen feministischen Aktivismus bestärken:

„Man spürt doch schon sehr stark, dass man nicht alleine ist dadurch. Und ich glaube, wenn ich den Podcast nicht hätte, wäre die Entmutigung viel schlimmer noch. Weil ich ja dadurch eigentlich in Kontakt gekommen bin mit vielen gleichdenkenden Personen. Oder ähnlich denkenden Personen." (M. Häcke, Interview, 11. März 2019)

Die Podcasterinnen haben die Erfahrung gemacht, dass die Nachrichten und Kommentare von Hörer:innen überwiegend positiv sind. Wie Mocigemba (ebd., S. 67) in einer früheren Podcasting-Studie, kommen die Interviewten zu dem Schluss, dass Hasskommentare oder Hassnachrichten bei Podcasts seltener auftreten als in anderen Medien. Sie begründen dies mit Eigenschaften des Mediums, da es einerseits länger dauere, einen Podcast anzuhören als einen Artikel zu überfliegen, und andererseits feministische Themen in Gesprächen besser erklärt werden und über die Stimme eine persönlichere Ebene geschaffen werden könne. Hier zeigt sich ein Potential des Mediums, öffentlich feministische Diskurse zu führen und Themen auszuhandeln, ohne die ständige Angst vor verletzenden und diskriminierenden Kommentaren:

„Da muss jemand Zeit investieren, um den anzuhören. Und wenn man das eigentlich nur machen will, um hinterher einen TrollKommentar oder einen Hass-Kommentar abzulassen, ist das ein bisschen zu viel Aufwand für einige von diesen klassischen Trollen, für die es irgendwie leichter ist, einen Blog-Artikel nach irgendwelchen Keywords zu scannen und dann daraus irgendwie ihren Hasskommentar zu bauen. Also ich würde schon sagen, dass ich da wahrscheinlich sehr viel weniger abkriege, als das feministische Blogger:innen z.B. kriegen. Aber das heißt nicht, dass das nicht passiert. Also auf jeden Fall gibt es da Kommentare in diese Richtung." 
(R. Görmann, Interview, 19. März 2019)

\section{Schluss}

Während die Zahl der Podcasthörer:innen sowie Podcastmacher:innen in Deutschland seit einigen Jahren steigt, gehen die interviewten Podcasterinnen davon aus, dass auch die Zahl und Bedeutung feministischer Podcasts zunehmen wird. Es bleibt an dieser Stelle offen, welche Netzwerk- und Reichweiteneffekte in der feministischen Podcastlandschaft in Zukunft entstehen. Klar ist, dass die Entwicklung dieses Mediums kein Selbstläufer sein wird, sondern den Mut und das Durchhaltevermögen der Podcaster:innen erfordert, die Podcasting als einen zentralen Bestandteil feministischer Öffentlichkeiten nutzen und gestalten wollen. Mit der vorliegenden Studie sollten erste Erkenntnisse über feministische Podcaster:innen gewonnen werden. Es hat sich gezeigt, dass Parallelen zu anderen unabhängigen Podcaster:innen und besonders zu feministischen Blogger:innen bestehen. Dennoch wird deutlich, dass die Kombination aus dem spezifischen Medium Podcasting und dem häufig emotional aufgeladenen Thema Feminismus ganz eigene Dynamiken entwickelt und neue Möglichkeiten eröffnet, aber auch neue Herausforderungen mit sich bringt. Gerade für die Arbeit feministischer Aktivist:innen und um Artikulationsräume für feministische Anliegen zu schaffen, scheint das Medium Podcast eine Chance zu bieten: das gesprochene Wort erleichtert Diskussionen und Aushandlungsprozesse, Gäst:innen können Podcasts bereichern und diverse Perspektiven einbringen, das Medium scheint sowohl für Produzent:innen als auch Hörer:innen offen und niedrigschwellig zu sein und dies unabhängig von großen Medienunternehmen. Durch das Podcasten entstehen Netzwerke und Communities und zuletzt scheint das Medium die Wahrscheinlichkeit von Hasskommentaren zu verringern. Dennoch wurde in den Interviews mit sechs feministischen Podcasterinnen deutlich, dass das Nutzen dieses Mediums für feministische Zwecke einen ständigen Reflexionsprozess erfordert und bestimmte Fragen aufwirft, die jede Podcaster:in für sich beantworten muss: Mit welcher Sprache und welchen Begrifflichkeiten werden die Diskussionen geführt? Welche Themen bekommen einen Raum in dem Podcast und welche Gäst:innen werden eingeladen? Wie können die Inhalte des Podcasts für alle Menschen zugänglich und verständlich sein? Wie kann der eigene Podcast finanziell unabhängig bleiben? Aber auch: Wie können Podcaster:innen unterstützt werden, die nicht die finanziellen und zeitlichen Ressourcen haben, um dauerhaft in ihrer Freizeit einen eigenen Podcast zu produzieren? Wie kann der Austausch in Netzwerken und Communities gefördert werden? Und wie kann eine positive Streitkultur entstehen?

In der vorliegenden Arbeit wurden feministische Podcasts als vernetzte Gegenöffentlichkeiten definiert. Die Interviews haben jedoch gezeigt, dass gerade unter feministischen Podcaster:innen und innerhalb der PodcastCommunities noch viel Potential zur Vernetzung besteht - und auch häufig 
der Wunsch, Podcasting als kollektive feministische Praxis zu stärken und dessen emanzipatorisches Potential gemeinsam zu nutzen. So müssten auch Podcaster:innen ohne besondere feministische oder Podcasting-Vorkenntnisse nicht alle Fragen neu beantworten und Podcasting könnte als feministisches Medium an gesellschaftlicher Relevanz und Durchsetzungskraft gewinnen. Gleichzeitig wurde deutlich, dass feministische Podcasts für die Gender Media Studies ein wichtiges neues Forschungsfeld darstellen. Die Entwicklung und der Bedeutungsgewinn dieses feministischen Mediums eröffnet eine Vielzahl an Ansatzpunkten für weitere qualitative Studien.

\section{Literatur}

Aigner, I. \& Schiff, A. (2019). Netzfeminismus und seine Grenzen. Wir Frauen, $37(1)$.

Attig, C. (2019). Mehr als ein Hobby? Deutschsprachige Podcaster*innen im Fokus psychologischer Forschung: Präsentation auf dem 36. Chaos Communication Congress. Leipzig. Verfügbar unter: https://www-user.tu-chemnitz. de/ attig/36C3_Podcaststudie.pdf

Baer, H. (2016). Redoing feminism: digital activism, body politics, and neoliberalism. Feminist Media Studies, 16(1), 17-34. https://doi.org/10.1080/ 14680777.2015.1093070

Bock, S. (2010). Frauennetzwerke: Geschlechterpolitische Strategie oder exklusive Expertinnennetze? In R. Becker \& B. Kortendiek (Hrsg.), Handbuch Frauen- und Geschlechterforschung (S. 878-886). Wiesbaden: VS Verlag für Sozialwissenschaften. https://doi.org/10.1007/978-3-322-99461-5_85

Bowman, J. (o. J.). Podcasts von und mit Frauen: Macht Platz, Hirsche? Verfügbar unter: https://www.podigee.com/de/blog/podcasts-von-und-mitfrauen/

boyd, danah. (2011). Social Network Sites as Networked Publics: Affordances, Dynamics, and Implications. In Z. Papacharissi (Hrsg.), A Networked Self (S. 39-58). New York; London: Routledge.

Butler, J. (1999). Gender Trouble: Feminism and the Subversion of Identity. New York: Routledge.

Bücker, T. (2014). Gestärkte Bewegungen, geschwächte Aktivistinnen? Das Dilemma des Onlineaktivismus. Feministische Studien, 32(1), 116-127. https://doi.org/10.1515/fs-2014-0113

Carstensen, T. (2019). Social Media: Zwischen Selbstpräsentation und Unsichtbarkeit, Empowerment und Sexismus. In J. Dorer, B. Geiger, B. Hipfl \& V. Ratković (Hrsg.), Handbuch Medien und Geschlecht. Wiesbaden: Springer VS. https://doi.org/10.1007/978-3-658-20712-0_79-1 
Clark-Parsons, R. (2017). Feminist Ephemera in a Digital World: Theorizing Zines as Networked Feminist Practice. Communication, Culture \& Critique, 10(4), 557-573. https://doi.org/10.1111/cccr.12172

Daniels, J. (2009). Rethinking Cyberfeminism(s): Race, Gender, and Embodiment. Women's Studies Quarterly, 37(1-2), 101-124. https://doi.org/10.1353/ wsq.0.0158

Dicker, R. C. \& Piepmeier, A. (Hrsg.). (2003). Catching a Wave: Reclaiming Feminism for the 21st Century. Boston: Northeastern University Press.

Diemand, V., Mangold, M. \& Weibel, P. (Hrsg.). (2007). Weblogs, Podcasting und Videojournalismus: Neue Medien zwischen demokratischen und ökonomischen Potenzialen. Hannover: Heise.

Doane, B., McCormick, K. \& Sorce, G. (2017). Changing methods for feminist public scholarship: lessons from Sarah Koenig's podcast Serial. Feminist Media Studies, 17(1), 119-121. https://doi.org/10.1080/14680777.2017.1261465

Drüeke, R. (2017). Feminismus im Netz - Strategien zwischen Empowerment und Angreifbarkeit. Feministische Studien, 35(1), 137-147. https://doi.org/10. 1515/fs-2017-0012

Drüeke, R. (2018). Digitale Medien: affirmative Geschlechterordnungen und feministische Interventionen. In B. Kortendiek, B. Riegraf \& K. Sabisch (Hrsg.), Handbuch interdisziplinäre Geschlechterforschung. Wiesbaden: Springer VS. https://doi.org/10.1007/978-3-658-12496-0_82

Drüeke, R. (2019). Digitale Öffentlichkeiten und feministische Protestkulturen. In J. Dorer, B. Geiger, B. Hipfl \& V. Ratković (Hrsg.), Handbuch Medien und Geschlecht. Wiesbaden: Springer VS. https://doi.org/10.1007/978-3-658-207120_42-1

Engelmann, K., Günther, F., Heise, N., Hohmann, F., Irrgang, U. \& Schmidt, S. (2010). Muslimische Weblogs: Der Islam im deutschsprachigen Internet. Berlin: Frank \& Timme.

Fields, E. (2011). Feminist Podcasting : Delivering Scholarship Outside the University Walls. Feminist Collections, 32(1), 26-27. https://doi.org/10.14288/ 1.0077927

Fotopoulou, A. (2016). Feminist Activism and Digital Networks: Between Empowerment and Vulnerability (Palgrave Studies in Communication for Social Change). London: Palgrave Macmillan. https://doi.org/10.1057/978-1-13750471-5

Fraser, N. (1992). Rethinking the Public Sphere: A Contribution to the Critique of Actually Existing Democracy. In C.J. Calhoun (Hrsg.), Habermas and the Public Sphere (S. 109-142). Cambridge: MIT Press.

Gaden, G. (2009). Podcasting: Thinking about new opportunities for pedagogy and activism. thirdspace, 9(1). 
Garrison, E. K. (2000). U.S. Feminism-Grrrl Style! Youth (Sub)Cultures and the Technologics of the Third Wave. Feminist Studies, 26(1), 141-170. https://doi.org/10.2307/3178596

Gunnarsson Payne, J. (2012). Feminist Media as Alternative Media? Theorising Feminist Media from the Perspective of Alternative Media Studies. In E. Zobl \& R. Drüeke (Hrsg.), Feminist Media (S. 55-72). Bielefeld: transcript. https://doi.org/10.14361/transcript.9783839421574.55

Gynnild, A. (2016). Applying Grounded Theory in Media Production Studies. In C. Paterson, D. Lee, A. Saha \& A. Zoellner (Hrsg.), Advancing Media Production Research (S. 115-130). London: Palgrave Macmillan.

Haidacher, C. (2014). Bewaffnet mit Kugelschreiber und Mikrofon. Medien als Werkzeug sozialer Entwicklung. Eine Studie. Wien: Frauensolidarität. Verfügbar unter: http://www.frauensolidaritaet.org/images/doku/medien_de.pdf

Hansen, H. (2013). Feminismus reloaded - das Weblog Mädchenmannschaft. In B. Kampmann, B. Keller, M. Knippelmeyer \& F. Wagner (Hrsg.), Die Frauen und das Netz (S. 149-158). Wiesbaden: Gabler Verlag. https://doi.org/10.1007/ 978-3-8349-4129-9_10

Harris, A. (2010). MIND THE GAP: Attitudes and Emergent Feminist Politics since the Third Wave. Australian Feminist Studies, 25(66), 475-484. https://doi.org/10.1080/08164649.2010.520684

Harris, A. (Hrsg.). (o. J.a). Next Wave Cultures: Feminism, Subcultures, Activism. New York; Abingdon: Routledge.

Harris, A. (o. J.b). Young women, late modern politics, and the participatory possibilities of online cultures. Journal of Youth Studies, 11(5), 481-495. https://doi.org/10.1080/13676260802282950

Keller, J. M. (2012). VIRTUAL FEMINISMS: Girls' blogging communities, feminist activism, and participatory politics. Information, Communication \& Society, 15(3), 429-447. https://doi.org/10.1080/1369118X.2011.642890

Keller, J. M. (2013). Still Alive and Kicking: Girl Bloggers and Feminist Politics in a Postfeminist Age. Dissertation. Austin: The University of Texas at Austin. Verfügbar unter: https://repositories.lib.utexas.edu/handle/2152/21560

Kempson, M. (2015). „My Version of Feminism“: Subjectivity, DIY and the Feminist Zine. Social Movement Studies, 14(4), 459-472. https://doi.org/10.1080/ 14742837.2014.945157

Klingner, S. (o. J.). Zwei Männer unterhalten sich. Verfügbar unter: https:// hauseins.fm/zwei-maenner-unterhalten-sich/

Knapp, G.-A. (2005). Intersectionality : ein neues Paradigma feministischer Theorie? ; Zur transatlantischen Reise von Race, Class, Gender. Feministische Studien, 23(1), 68-81. https://doi.org/10.25595/618 
Knappe, H. \& Lang, S. (2014). Between whisper and voice: Online womens movement outreach in the UK and Germany. European Journal of Women's Studies, 21(4), 361-381. https://doi.org/10.1177/1350506814541643

Lauber, A., Wagner, U. \& Theunert, H. (2007). Internetradio und Podcasts - neue Medien zwischen Radio und Internet: Eine explorative Studie zur Aneignung neuer Audioangebote im Auftrag der Bayerischen Landeszentrale für neue Medien (BLM). München. Verfügbar unter: https://www.jff. de/veroeffentlichungen/detail/ergebnisbericht-zur-explorationsstudieinternetradio-und-podcasts-neue-medien-zwischen-radio-und-int/

Lenz, I. (2010). Intersektionalität: Zum Wechselverhältnis von Geschlecht und sozialer Ungleichheit. In R. Becker \& B. Kortendiek (Hrsg.), Handbuch Frauenund Geschlechterforschung (S. 158-165). Wiesbaden: VS Verlag für Sozialwissenschaften.

Lenz, I. (2018). Von der Sorgearbeit bis \#MeToo. Aktuelle feministische Themen und Debatten in Deutschland. Aus Politik und Zeitgeschehen, 68(17), 2027.

Llinares, D., Fox, N. \& Berry, R. (Hrsg.). (2018). Podcasting: New Aural Cultures and Digital Media. Cham: Palgrave Macmillan.

Lünenborg, M. \& Meier, T. (2018). Interviews als Form der Produktionsanalyse: Ein Praxiseinblick in die Forschung im Kontext der Gender Media Studies. In C. Pentzold, A. Bischof \& N. Heise (Hrsg.), Praxis Grounded Theory (S.169-189). Wiesbaden: Springer VS. https://doi.org/10.1007/978-3-658-15999-3_8

Markman, K. M. (2012). Doing radio, making friends, and having fun: Exploring the motivations of independent audio podcasters. New Media \& Society, 14(4), 547-565. https://doi.org/10.1177/1461444811420848

Markman, K. M. \& Sawyer, C. E. (2014). Why Pod? Further Explorations of the Motivations for Independent Podcasting. Journal of Radio \& Audio Media, 21(1), 20-35. https://doi.org/10.1080/19376529.2014.891211

Millette, M. (2011). Independent Podcasting as a Specific Online Participative Subculture: A Case Study of Montreal's Podcasters. AoIR Selected Papers of Internet Research, 1. https://doi.org/10.5210/spir.v1io.9111

Mocigemba, D. (2006). Warum sie selber senden: eine Typologie von Sendemodi im Podcasting. kommunikation@gesellschaft, 7(3), 1-29.

Mocigemba, D. (2007). Sechs Podcast-Sendetypen und ihre theoretische Verortung. In S. Kimpeler, M. Mangold \& W. Schweiger (Hrsg.), Die digitale Herausforderung (S. 61-76). Wiesbaden: VS Verlag für Sozialwissenschaften. https://doi.org/10.1007/978-3-531-90649-2\{|textunderscore \}6

Müller, K. F. (2018). Theoretisches Kodieren von Interviewmaterial. Medienaneignung mit der Grounded Theory induktiv analysieren. In C. Pentzold, A. Bischof \& N. Heise (Hrsg.), Praxis Grounded Theory (S. 149-168). Wiesbaden: Springer VS. https://doi.org/10.1007/978-3-658-15999-3_7 
Novoselova, V. \& Jenson, J. (2018). Authorship and professional digital presence in feminist blogs. Feminist Media Studies, 19(2), 257-272. https://doi.org/10.1080/14680777.2018.1436083

Piepmeier, A. (2009). Girl zines: Making media, doing feminism. New York: New York University Press.

Pruchniewska, U. M. (2018). Branding the self as an „authentic feminist": negotiating feminist values in post-feminist digital cultural production. Feminist Media Studies, 18(5), 810-824. https://doi.org/10.1080/ 14680777.2017.1355330

Reuter, M. (о. J.). Mehr als 500 Podcasts von und mit Frauen in Deutschland. Verfügbar unter: https://netzpolitik.org/2017/mehr-als-500-podcasts-vonund-mit-frauen-in-deutschland/

Richardson, S. \& Green, H. (2018). Talking women/women talking: the feminist potential of podcasting for modernist studies. Feminist Modernist Studies, 1(3), 282-293. https://doi.org/10.1080/24692921.2018.1505818

Sadowski, H. (2016). From \#aufschrei to hatr.org: digital-material entanglements in the context of German digital feminist activisms. Feminist Media Studies, 16(1), 55-69. https://doi.org/10.1080/14680777.2015.1093090

Schmidt, J. (2007). Stabilität und Wandel von Weblog-Praktiken: Erste empirische Befunde. In S. Kimpeler, M. Mangold \& W. Schweiger (Hrsg.), Die digitale Herausforderung (S. 51-60). Wiesbaden: VS Verlag für Sozialwissenschaften. https://doi.org/10.1007/978-3-531-90649-2_5

Schmincke, I. (2019). Die Neue Frauenbewegung in den Medien. In J. Dorer, B. Geiger, B. Hipfl \& V. Ratković (Hrsg.), Handbuch Medien und Geschlecht. Wiesbaden: Springer VS. https://doi.org/10.1007/978-3-658-20712-0_40-1

Shaw, F. (2012). Discursive politics online: political creativity and affective networking in Australian feminist blogs. Dissertation. Sydney: University of New South Wales. Verfügbar unter: https://www.academia.edu/2031938/Discursive_ politics_online_political_creativity_and_affective_networking_in_ Australian_feminist_blogs_PhD_Thesis

Steiner, L. (1992). The History and Structure of Women's Alternative Media. In L.F. Rakow (Hrsg.), Women Making Meaning (S. 121-143). New York: Routledge.

Steiner, L. (2012). Using New Technologies to Enter the Public Sphere, Second Wave Style. In E. Zobl \& R. Drüeke (Hrsg.), Feminist Media (S. 182-196). Bielefeld: transcript. https://doi.org/10.14361/transcript.9783839421574.182

Stier, S. (2017). Internet und Regimetyp: Netzpolitik und politische OnlineKommunikation in Autokratien und Demokratien. Wiesbaden: Springer VS.

Stoltenhoff, A.-K. \& Raudonat, K. (2018). Digitalisierung (mit)gestalten - was wir vom Cyberfeminismus lernen können: Strategien und Ansätze einer aktivierenden Perspektive auf Informations- und Kommunikationstechnologien 
im 21. Jahrhundert. GENDER - Zeitschrift für Geschlecht, Kultur und Gesellschaft, 10(2), 128-142. https://doi.org/10.3224/gender.v10i2.09

Strauss, A. L. \& Corbin, J. M. (1998). Basics of qualitative research: Techniques and procedures for developing grounded theory (2. Auflage). Thousand Oaks: Sage.

Strübing, J. (2018). Grounded Theory: Methodische und methodologische Grundlagen. In C. Pentzold, A. Bischof \& N. Heise (Hrsg.), Praxis Grounded Theory (S. 27-52). Wiesbaden: Springer VS. https://doi.org/10.1007/978-3658-15999-3\{|textunderscore $\} 2$

Thiessen, B. (2010). Feminismus: Differenzen und Kontroversen. In R. Becker \& B. Kortendiek (Hrsg.), Handbuch Frauen- und Geschlechterforschung (S. 3744). Wiesbaden: VS Verlag für Sozialwissenschaften. https://doi.org/10.1007/ 978-3-531-91972-0\{|textunderscore $\} 4$

Tiffe, R. \& Hoffmann, M. (2017). Taking up sonic space: feminized vocality and podcasting as resistance. Feminist Media Studies, 17(1), 115-118. https://doi.org/10.1080/14680777.2017.1261464

Walgenbach, K. (2012). Intersektionalität - eine Einführung. Verfügbar unter: http://portal-intersektionalitaet.de/uploads/media/WalgenbachEinfuehrung.pdf

Washick, B. M. (2016). Strange Spaces \& Stranger Sensibilities: Feminist CounterPublicity in the Digital Age. Dissertation. Ann Arbor: University of Michigan. Verfügbar unter: http://hdl.handle.net/2027.42/120905 